J. Clin. Chem. Clin. Biochem.

Vol. 15, 1977, pp. 619-624

\title{
Biochemische Befunde in Zystenflüssigkeiten zystisch degenerierter euthyreoter Strumen
}

\author{
Von G. Galvan, H. J. Gibitz, D. Hauch und F. Maier
}

Aus der Nuklearmedizinischen Station (Leiter Dr. G. Galvan) und dem chemischen Zentrallaboratorium (Vorstand Prim. Dr. H. J. Gibitz) der Landeskrankenanstalten und aus dem Zentrum für elektronische Datenverarbeitung (Vorstand Prof. Dr. P. Zinterhof) der Universität Salzburg

(Eingegangen am 12. Mai/3. August 1977)

Żusammenfassung: Untersuchungen an Zystenflüssigkeiten und Seren von Patienten mit zystisch degenerierten euthyreoten Strumen ergaben für die meisten untersuchten chemischen Substanzen signifikante Konzentrationsunterschiede zwischen Zystenflüssigkeit und Serum. In der gelben Zystenflüssigkeit finden sich durchwegs niedrigere, in brauner Zystenflüssigkeit meist höhere Konzentrationen als in den zugehörigen Seren. Diffusions- und Resorptionsvorgänge, autolytische und metabolische Prozesse und für Schilddrüsenhormone auch eine aktive Inkretion durch wandbildendes Schilddrüsengewebe sind Ursache der Konzentrationsunterschiede.

\section{Biochemical data in the fluids of cystic degenerated euthyroid goiters}

Summary: Examinations of sera and cyst fluids in patients with cystic degenerated euthyroid goiters showed significant differences between the values of the cyst fluids and sera for almost all of the chemical substances. In the brown cyst fluids there are mostly higher values compared with the appropriate sera. Autolysis, diffusion and resorption, metabolic changes and, in the case of thyroid hormones, active incretion by wall-forming thyroid tissue, are the reasons for these differences in the concentrations.

\section{Einführung}

Von den im Radiojod- bzw. 99 m Tc-Szintigramm „kalten", also hypofunktionellen Strumaknoten sind $22,6 \%$ durch zystische Degeneration des Strumaknotens bedingt (1). Die Farbe der Zystenflüssigkeit ist zumeist gelb, oder in zeitlicher Abhängigkeit von erfolgten Blutungen rot oder schokoladebraun bis hellbraun.

In der klinischen Diagnostik wird die Aufdeckung einer Strumapseudozyste im Rahmen der Feinnadelpunktion zur zytologischen Abklärung kalter Strumaknoten mit Erleichterung zur Kenntnis genommen, da die Malignominzidenz bei kalten Strumaknoten $1: 25$, bei Vorliegen von Pseudozysten dagegen $1: 343$ beträgt (1).

Uber die Zystenflüssigkeit selbst liegen bisher nur spärliche Angaben vor ${ }^{1}$ ): so wird gegenüber dem Serum eine Verminderung von Protein, Cholesterin und Cholinesterase bei Erhöhung der Lactatdehydrogenase erwähnt

1) Die Literatursuche wurde über den medizinischen Literatur informationsdienst LID, Österr. Bundesinstitut für Gesundheitswesen, durchgefựuț.
(2). Untersuchungen über die Proteinzusammensetzung der Zystenflüsigkeit in der Papierelektrophorese (3) ergaben ein dem Serum ähnliches Proteinmuster bei unterschiedlichen morphologischen Bildern der Zystenwand.

\section{Material und Methoden}

Untersucht wurden Seren und durch Feinnadelpunktion gewonnene Zystenflüssigkeiten von 82 Strumapseudozysten bei 82 Patienten mit euthyreoten Strumen. Die Diagnose der Euthyreose wurde durch klinișches Bild, Achillessehnenreflexzeit, Radiojod-bzw. 99 m Tc-Szintigramm, sowie durch Bestimmung folgender in vitro-Tests gestellt: effektiver Thyroxinindex (ETI Bio-Rad), Gesamttrijodthyronin (T3 RIA Le Petit), Gesamtthyroxin (T4-RIA Abbott), thyreotropes Hormon (TSH RIA-Mat Byck Mallinckrodt) vor und 30 Minuten nach $200 \mu \mathrm{g}$ TRH (Relefact ${ }^{\circledR}$ Hoechst).

Die Feinnadelpunktion der Strumapseudozysten wurde in typischer Weise durchgeführt (4).

Die Blutabnahmen erfolgten nüchtern, zwischen 7 und $10 \mathrm{Uhr}$, Blut und Zystenflüssigkeit wurden unmittelbar nach der Abnahme zentrifugiert und innerhalb von 2 Stunden verarbeitet oder bis zur Analyse bei $-20^{\circ} \mathrm{C}$ eingefroren. Zystenflüssigkeiten wurden zum Ausschluß eines Malignoms zytologisch untersucht. 
Chemische Bestimmungsmethoden

Die Präzision der Bestimmungsmethoden für die einzelnen chemischen Parameter, die aus Seren und Zysteninhalten bestimmt wurden, erfolgt durch Angabe des Variationskoeffizienten (VK) bezogen auf die Präzision von Tag zu Tag. Normbereiche im Serum (NW) und VK sind für die einzelnen Bestimmungsmethoden in Klammern angegeben.

1. Bilirubin (NW bis $17 \mu \mathrm{mol} / 1$, VK $2,40 \%$ ).

Bestimmung des Gesamtbilirubin im ,Technicon Auto-

Analyzer II" nach Jendrassik \& Gróf (5).

2. Glucose (NW 4,44-5,83 mmol/1, VK 2,89\%).

Bestimmung mit Testkombination „Blutzucker-GOD-Perid“ Boehringer Mannheim Nr. 124.028, nach Enteiweißung mit Uranylacetat, Fotometer Eppendorf, Filter $\mathrm{Hg} 578 \mathrm{~nm}$.

3. Triglyceride (NW 0,80-1.94 mmol/1, VK 9,37\%).

Enzymatische Bestimmung des Gesamtglycerins nach Verseifung mit Kalilauge, mit Biochemia Testkombination „Neutralfette“ Boehringer-Mannheim Nr. 125.032, Fotometer Eppendorf, Filter $\mathrm{Hg} 365 \mathrm{~nm}$.

4. Cholesterin (NW 4,13-6,21 mmol/1, VK 3,18\%).

Bestimmung des Gesamt-Cholesterins im „Technicon AutoAnalyzer II" nach Liebermann-Burchard (6).

5. Magnesium (NW 0,78-1,03 mmol/l, VK 5,34\%). Bestimmung mittels Xylidylblau-Reaktion mit Merckotest Magnesium“ Nr. 3338, Fotometer Eppendorf, Filter Hg $492 \mathrm{~nm}$.

6. Calcium (NW 2,25-2,47 mmol/l, VK 2,58\%).

Bestimmung mit der Testpackung „Calcium Rapid State

Kit" Pierce Chem. Comp., Rockford, Illinois, Nr. 40895.

Farbreaktion mit Methylthymolblau in alkalischem Milieu, Fotometer Eppendorf, Filter $\mathrm{Hg} 623 \mathrm{~nm}$.

7. Anorg. Phosphor (NW 0,65-1,29 mmol/l, VK 3,50\%). Nach Enteiweißung mit Trichloressigsäure Messung des blauen Farbkomplexes, der durch Reaktion des Phosphats mit Ammoniummolybdat und Eisenammcniumsulfat entsteht (7), Fotometer Eppendorf, Filter Hg $578 \mathrm{~nm}$.

8. Eisen (NW 14,3-26,9 $\mu \mathrm{mol} / 1$, VK $3,47 \%$ ).

Das aus Transferrin freigesetzte Eisen wird im „Technicon Auto-Analyzer II" als Ferrozin-Komplex fotometrisch bestimmt (8).

9. Kupfer (NW 11,0-20,5 $\mu \mathrm{mol} / 1, \mathrm{VK} 4,17 \%$ ).

Bestimmung mit der Testpackung „Kupfer im Serum und Urin“, Dr. Heinz Haury, Chemische Fabrik 8 München, Fotometer Eppendorf, Filter $\mathrm{Hg} 492 \mathrm{~nm}$.

10. Kalium (NW 4,1-5,4 mmol/1, VK 1,29\%). Flammenfotometrische Bestimmung im ,Technicon Analyzer SMA 6/60" (9).

11. Natrium (NW $135-152 \mathrm{mmol} / \mathrm{l}, \mathrm{VK} \quad 0,62 \%$ ).

Flammenfotometrische Bestimmung im „Technicon Analyzer SMA 6/60" (9).

12. Chlorid (NW 98-107 mmol/1, VK 0,78\%).

Fotometrische Bestimmung im ,Technicon Analyzer SMA $6 / 60 "(10)$.

13. Hydrogencarbonat (NW 20-26 mmol/1, VK 5,64\%). Fotometrische Bestimmung im ,Technicon Analyzer SMA 6/60". Farbreaktion mit Kresolrot bei pH 9,2 (11).

14. Harnstoff (NW bis $6,7 \mathrm{mmol} / 1$, VK $1,95 \%$ ). Bestimmung mit dem ,Technicon Analyzer SMA 6/60“, Diacetylmonoximmethode (12).

15. Creatinin (NW bis $106 \mathrm{mmol} / \mathrm{l}$, VK 3,06\%). Bestimmung im „Technicon Analyzer SMA 6/60“, JafféReaktion im Dialysat (13).

16. Harnsäure (NW 178,4-389,0 $\mu \mathrm{mol} / 1$, VK 2,12\%). Enzymatische Bestimmung mit Biochemia Testkombination „Uricaquant", Boehringer-Mannheim Nr. 124.761, LKB Absorptiometer 7400, Wellenlänge $405 \mathrm{~nm}$.

17. Elektrophorese

Bestimmung des Gesamtprotein mittels Biuret-Reaktion im Technicon Auto-Analyzer I" (14). Mikrozonen-Elektrophorese auf Celluloseacetatfolien (Mikrophor, Boskamp:

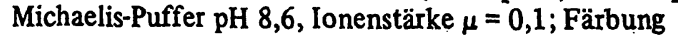
Amidoschwarz). Auswertung mit „Elphomat I" (Bender und Hobein, München). Umrechnung der Relativwerte in $\mathrm{g} / \mathrm{l}$, bezogen auf das Gesamtprotein.

NW im Serum: Gesamtprotein $65-85 \mathrm{~g} / 1$. Albumine 35-56 g/l. $\alpha_{1}$-Globuline 1,3-4,3 $\mathrm{g} / \mathrm{l}$. $\alpha_{2}$-Globuline 3,2$9,4 \mathrm{~g} / \mathrm{l}$. $\beta$-Globuline 5,8-12,8 $\mathrm{g} / \mathrm{l}, \gamma$-Globuline $7,2-17 \mathrm{~g} / \mathrm{l}$.
18. Alkalische Phosphatase (EC 3.1.3.1) (NW 20-48 U/l, VK 6,75\%).

Bestimmung mit „Biochemia Testkombination“ BoehringerMannheim Nr. $123.889,30$ Minuten-Methode bei $37^{\circ} \mathrm{C}$.

19. Saure Phosphatase (EC 3.1.3.2). (NW bis $13 \mathrm{U} / 1$, VK 7,44\%). Bestimmung der „gesamten sauren Phosphatase" mit „Biochemia Testkombination" Boehringer-Mannheim Nr. 125.008, 30 Minuten Methode bei $37^{\circ} \mathrm{C}$.

20. Alaninaminotransferase (EC 2.6.1.2). (NW bis $12 \mathrm{U} / 1$, VK $6,0 \%)$.

Reagenzien Boehringer-Mannheim GPT Nr. 124.656, Eppendorf Enzymautomat 5010, Filter Hg 334 nm, nicht optimierter UV-Test bei $25^{\circ} \mathrm{C}$. (15).

21. Aspartataminotransferase (EC 2.6.1.1), (NW bis $12 \mathrm{U} / \mathrm{l}$, VK 5,62\%).

Reagenziien Boehrịnger-Mannheim, GOT Nr. 124.508, Eppendorf Enzymautomat 5010, Filter $\mathrm{Hg} 334 \mathrm{~nm}$, nicht optimierter UV-Test bei $25^{\circ} \mathrm{C}(15)$.

22. Lactatdehydrogenase (EC 1.1.1.27), (NW 80-240 U/l, Ví $7,90 \%)$.

Reagenzien Boehringer-Mannheim, LDH Nr. 124.915. Eppendorf Enzymautomat 5010, optimierter UV-Test, Filter $\mathrm{Hg} 334 \mathrm{~nm}, 25^{\circ} \mathrm{C}$.

23. $\alpha$-Amylase (EC 3.2.1.1), (NW bis 1000 U/l, VK 5,77\%). Amyloklastische Methode (16), Substrat $1 \mathrm{~g} / \mathrm{I}$ Stärkelösung, fotometrische Messung der Intensität des blauen Jod-StärkeKomplexes, Fotometer Eppendorf, Filter $\mathrm{Hg} 578 \mathrm{~nm}, 37^{\circ} \mathrm{C}$.

24. $\alpha$-Hydroxybutyrat-Dehydrogenase (NW bị $140 \mathrm{U} / \mathrm{l}$, VK 5,67\%).

Bestimmung mit „Biochemia Testkombination $\alpha$-HBDHopt". Boehringer-Mannheim Nr. 124.818. Fotometer Eppendorf, Filter $\mathrm{Hg} 365 \mathrm{~nm}, 25^{\circ} \mathrm{C}$.

25. $\gamma$-Glutamyl-Transpeptidase (EC 2.3.2.2), NW $4=28 \mathrm{U} / 1$, VK 4,90\%).

Bestimmung mit „Biochemia Testkombination $\gamma$-GT MonoTest Boehringer-Mannheim Nr. 125.938. Fotometer Eppendorf, Filter $\mathrm{Hg} 405 \mathrm{~nm}, 25^{\circ} \mathrm{C}$.

26. Cholinesterase (EC 3.1.1.8), (NW 3000-8000 U/l, VK 2,33\%).

Bestimmung mit „Merck-1-Test Cholinesterase kinetisch", Fa. E. Merck-Darmstadt Nr. 3325, Substrat Butyrylthiocholin, Fotometer Eppendorf, Filter $\mathrm{Hg} 405 \mathrm{~nm}, 25^{\circ} \mathrm{C}$.

27. Proteingebundenes Jod (NW 236-591 nmol/1, VK 3,42\%). Bestimmung als Gesamtjod im "Technicon Auto-Analyzer" (17).

Radioimmunologische Bestimmungsmethoden

28. $L$-Trijodthyronin $\mathrm{T}_{3}$ (NW 0,80-1,80 $\mu \mathrm{g} / 1$, Interassayvarianz $15,8 \%$, Intraassayvarianz $4,1 \%$ ) mit dem Testbesteck $\mathrm{T}_{3}$ RIA Abbott.

29. $L$-Thyroxin $\mathrm{T}_{4}$ (NW 35-120 $\mu \mathrm{g} / \mathrm{l}$, Interassayvarianz $10,3 \%$, Intraassayvarianz 4,5\%), mit dem Testbesteck $\mathrm{T}_{4}$ RIA Abbott.

30. Reverse-Trijodthyronin $\mathrm{r}-\mathrm{T}_{3}$ (NW 90-350 ng/l, Interassay: varianz $10,4 \%$, Intraassayvarianz $7,6 \%$ ) mit dem Testbesteck „Reverse T 3 RIA Serono“ Code 1834.

31. Insulin (NW 2,5-25 mU/I, Interassayvarianz 9,7\%, Intraassayvarianz 6,4\%) mit dem Le Petit Insulin-Kit.

32. Wachstumshormon (NW $<3 \mu \mathrm{g} / \mathrm{L}$ in Ruhe, Interassayvarianz 11,4, Intraassayvarianz 7,2\%) mit dem HGH RIA Kit Abbott.

\section{Statistische Bearbeitung}

Auf Grund der geringen Stichprobenumfänge wurden verteilungsfreie Testverfahren herangezogen, deren Effizienz im Falle normalverteilter Daten jener von parametrischèn Verfahren nahekommt.

1. Lokationsvergleiche zweier abhängiger Stichproben: Zur Prüfung der Hypothese, daß die Mediane der beiden Stichproben gleich sind, wurde der Wilcoxon matched pairs signed ranks Test (18) eingesetzt.

2. Korrelationsstatistische Untersuchungen:

Die Prüfung von korrelativen Zusammenhängen erfolgte mittels des Rang-Korrelationskoeffizienten von Spearman (19). 
3. Lokationsvergleiche zweier unabhängiger Stichproben: Zur Prüfung der Hypothese, daß die Verteilungsfunktionen zweier unabhängiger Stichproben gleich sind - also auch die Mediane - wurde der U-Test von Wilcoxon, Mann \& Whitney herangezogen. Die angegebenen Testgrößen wurden nach $1 . c$. (20) bestimmt. Für den exakten Test finden sich Tabellen in 1.c. (21).

Die Berechnungen wurden mittels der Programme BMDP2D und BMDP3S (HSCF der University of California, Los Angeles (20)) auf einer IBM 370/145 in Zusammenarbeit mit dem Landesrechenzentrum Salzburg durchgeführt.

\section{Ergebnisse}

\section{Braune Zysten (Táb. 1)}

In braunen Zysten sind gegenüber den Serumwerten Lactatdehydrogenase, $\alpha$-Hydroxybutyratdehydrogenase, Eisen, Harnsäure, $L$-Trijodthyronin und Reverse-Trijodthyronin $(\alpha=0,001), \alpha$-Amylase, proteingebundenes Jod, $L$-Thyroxin und Protein $(\alpha=0,01)$, saure Phos- phatase, Aspartataminotransferase und Albumin $(\alpha=$ $0,05)$ signifikant erhöht.

Gegenüber dem Serum vermindert sind Glucose, Hydrogencarbonat und Insulin $(\alpha=0,001)$, alkalische Phosphatase, Cholinesterase, Triglyceride und Wachstumshormon $(\alpha=0,01)$.

Cholesterin, Alaninaminotransferase, $\alpha_{1}-\alpha_{2}$-Globulinkomplex, $\beta$ - und $\gamma$-Globuline, sowie Kupfer, Calcium, Kalium, Natrium, Chlorid, Harnstoff und Creatinin sind in Zystenflüssigkeiten und Seren im Median nicht signifikant unterschieden. Bilirubin, $\gamma$-Glutamyltranspeptidase und Magnesium konnten wegen der Eigenfarbe der Zystenflüssigkeit nicht bestimmt werden.

$Z$ wischen Zystenflüssigkeiten und Seren findet sich für Harnstoff $(\alpha=0,001)$, Wachstumshormon $(\alpha=0,01)$, Hydrogencarbonat, Creatinin, Insulin und $L$-Thyroxin eine signifikante Korrelation $(\alpha=0,05)$. Für die übrigen Parameter findet sich statistisch keine Abhängigkeit der Zystenwerte von den Serumwerten.

Tab. 1. Konzentrationen in Zystenflüssigkeiten und Seren von Patienten mit braunen Strumapseudozysten ${ }^{+}$).

$\mathbf{n}=$ Anzahl der untersuchten Patienten

$\overline{\mathbf{x}}=$ Mittelwert

$s=$ Standardabweichung

$\mathrm{M}=$ Median

\begin{tabular}{|c|c|c|c|c|c|c|c|c|}
\hline \multirow[t]{2}{*}{ Stoff } & \multirow{2}{*}{$\begin{array}{l}\text { Meß- } \\
\text { größe }\end{array}$} & \multirow[t]{2}{*}{$\mathbf{n}$} & \multicolumn{3}{|l|}{ Zyste } & \multicolumn{3}{|l|}{ Serum } \\
\hline & & & $\overline{\mathbf{x}}$ & $\pm s$ & M & $\overline{\mathbf{x}}$ & $\pm s$ & $\mathbf{M}$ \\
\hline Glucose & $\mathrm{mmol} / 1$ & 10 & 0,93 & 1,25 & 0,06 & 4,30 & 0,6 & 4,41 \\
\hline Triglyceride & $\mathrm{mmol} / \mathrm{l}$ & 10 & 0,43 & 0,18 & 0,39 & 1,35 & 0,58 & 1,15 \\
\hline Cholesterin & $\mathrm{mmol} / \mathrm{l}$ & 10 & 6,30 & 1,82 & 6,10 & 5,59 & 1,38 & 5,09 \\
\hline Calcium & $\mathrm{mmol} / \mathrm{l}$ & 9 & 2,64 & 0,40 & 2,59 & 2,42 & 0,07 & 2,45 \\
\hline anorg. Phosphor & $\mathrm{mmol} / 1$ & 9 & 0,68 & 0,32 & 0,71 & 0,94 & 0,10 & 1,00 \\
\hline Eisen & $\mu \mathrm{mol} / \mathrm{l}$ & 8 & 111,2 & 49,1 & 103,9 & 20,7 & 3,5 & 20,2 \\
\hline Kupfer & $\mu \mathrm{mol} / \mathrm{l}$ & 5 & 10,1 & 5,4 & 11,6 & 19,3 & 8,7 & 17,8 \\
\hline Kalium & $\mathrm{mmol} / \mathrm{l}$ & 9 & 4,20 & 0,30 & 4,20 & 4,60 & 0,40 & 4,50 \\
\hline Natrium & $\mathrm{mmol} / \mathrm{l}$ & 9 & 140,0 & 5,4 & 140,0 & 139,5 & 3,7 & 139,0 \\
\hline Chlorid & $\mathrm{mmol} / \mathrm{l}$ & 9 & 100,0 & 5,6 & 101,0 & 102,1 & 2,1 & 102,0 \\
\hline Hydrogencarbonat & $\mathrm{mmol} / \mathrm{l}$ & 9 & 16,9 & 4,7 & 16,0 & 23,2 & 3,2 & 25,0 \\
\hline Harnstoff & $\mathrm{mmol} / 1$ & 9 & 5,82 & 1,55 & 3,83 & 5,68 & 1,43 & 5,00 \\
\hline Creatinin & $\mu \mathrm{mol} / \mathrm{l}$ & 8 & 88,4 & 8,8 & 88,4 & 88,4 & 8,8 & 88,4 \\
\hline Harnsäure & $\mu \mathrm{mol} / \mathrm{l}$ & 8 & 398,5 & 113,0 & 422,3 & 261,7 & 53,5 & 261,7 \\
\hline Protein & $\mathrm{g} / 1$ & 11 & 94,5 & 27,9 & 85,0 & 72,8 & 4,6 & 71,0 \\
\hline Albumine & $\mathrm{g} / \mathrm{l}$ & 11 & 52,9 & 16,3 & 49,1 & 41,7 & 3,1 & 42,8 \\
\hline$\alpha_{1}$-Globuline & $\mathrm{g} / 1$ & 4 & 2,5 & 0,7 & 2,4 & 2,5 & 0,4 & 2,2 \\
\hline$\alpha_{2}$-Globuline & $g / 1$ & 4 & 3,3 & 1,9 & 3,0 & 7,3 & 1,0 & 7,5 \\
\hline$\beta$-Globuline & $g / 1$ & 9 & 10,4 & 4,4 & 10,1 & 7,9 & 1,1 & 7,8 \\
\hline $\boldsymbol{\gamma}$-Globuline & $g / 1$ & 11 & 16,7 & 13,2 & 13,4 & 13,4 & 3,0 & 12,4 \\
\hline$\alpha_{1}+\alpha_{2}$-Globuline & $\mathrm{g} / \mathrm{l}$ & 10 & 15,6 & 11,8 & 10,8 & 9,6 & 1,1 & 9,4 \\
\hline Alkalische Phosphatase & $\mathrm{U} / 1$ & 10 & 18,1 & 8,6 & 18,0 & 39,4 & 13,4 & 35,0 \\
\hline Saure Phosphatase & $\dot{U} / 1$ & 8 & 41,4 & 32,1 & 44,5 & 9,1 & 1,5 & 9,3 \\
\hline Alaninaminotransferase & $\mathrm{U} / 1$ & 8 & 12,2 & 16,3 & 6,0 & 7,6 & 6,0 & 5,0 \\
\hline Aspartataminotransferase & $\mathrm{u} / \mathrm{i}$ & 8 & 20,1 & 15,8 & 9,0 & 7,9 & 1,8 & 8,0 \\
\hline Lactatdehydrogenase & $\mathrm{U} / \mathrm{i}$ & 10 & 2586,0 & 1574,0 & 2325,0 & 201,2 & 62,7 & 191,0 \\
\hline$\alpha$-Hydroxybutyratdehydrogenase & $\mathbf{U} / \mathbf{1}$ & 9 & 1177,0 & 871,0 & 1330,0 & 103,7 & 21,2 & 100,0 \\
\hline$\alpha$-Amylase & $\mathrm{U} / 1$ & 10 & 4582,0 & 5971,7 & 2355,0 & 523,0 & 234,0 & 520,0 \\
\hline Cholinesterase & U/1 & 9 & 2546,7 & 1336,0 & 1960,0 & 5655,6 & 1287,9 & 5430,0 \\
\hline Proteingebundenes Jod & nmoli/l & 13 & 6051,8 & 9519,0 & 1607,5 & 504,3 & 86,7 & 488,6 \\
\hline$L$-Trijodthyronin & $\mu \mathrm{g} / 1$ & 15 & 3,42 & 2,30 & 2,60 & 1,53 & 0,34 & 1,45 \\
\hline Reverse-Trijodthyronin & $\mathrm{ng} / \mathrm{l}$ & 15 & 620 & 460 & 540 & 240 & 110 & 220 \\
\hline L-Thyroxin & $\mu \mathrm{g} / \mathrm{l}$ & 15 & 156 & 122 & 124 & 80 & 20 & 81 \\
\hline Insulin & $\mathrm{mU} / 1 \mathrm{l}$ & 23 & 1,8 & 2,5 & 0,0 & 9,8 & 9,9 & 7,0 \\
\hline Wạchstumshormon & $\mu \mathrm{g} / \mathrm{i}$ & $2 \dot{3}$ & 1,7 & 1,5 & 1,1 & 3,7 & 3,5 & 1,9 \\
\hline
\end{tabular}

${ }^{+}$) Bilirubin, Magnesium und $\gamma$-Glutamyltranspeptidase konnten wegen störender Farbreaktionen in der braunen Zystenflüssigkeit nicht bestimmt werden. 
Gelbe Zysten (Tab. 2)

In gelben Zystenflüssigkeiten zeigen die Zystenwerte gegenüber den Serumwerten für Cholinesterase, Triglyceride, Cholesterin, Kupfer, Protein, Albumin, $\alpha_{2}$, $\beta$ - und $\gamma$-Globulin $(\alpha=0,001)$, saure Phosphatase, Aspartataminotransferase, Natrium, $\alpha_{1}-\alpha_{2}$-Globulinkomplex $(\alpha=0,01)$, Lactatdehydrogenase, Magnesium und $L$-Thyroxin $(\alpha=0,05)$ eine signifikante Erniedrigung. Die übrigen Parameter sind nicht signifikant unterschieden.

Die Zystenwerte korrelieren mit den Serumwerten für proteingebundenes Jod, Harnsäure, $L$-Thyroxin, $\alpha_{2}$ und $\beta$-Globuline $(\alpha=0,001)$, saure Phosphatase, Aspartataminotransferase, $\alpha$-Amylase, $\gamma$-Glutamyltranspeptidase, Cholesterin, Hamstoff, $\gamma$-Globuline und Wachstumshormon $(\alpha=0,01)$, Cholinesterase, Kupfer, Creatinin und $\alpha_{1}-\alpha_{2}$-Globulinkomplex $(\alpha=0,05)$. Für die übrigen Parameter finden sich keine Korrelationen zwischen Zysten- und Serumwerten.

\section{Gegenüberstellung braune Zystè - gelbe Zyste}

Während sich die Serumwerte von Patienten mit braunen und gelben Zysten für die einzelnen Parameter im Median nicht signifikant unterscheiden, findet sich zwischen gelber und brauner Żystenflüssigkeit für saure Phosphatase, Aspartataminotransferase, Lactatdehydrogenase, Glucose, Cholesterin, Eisen und Protein ( $\alpha=$ $0,001), \alpha$-Amylase, proteingebundenes Jod, Albumine, $\beta$-Globuline, $L$-Trijodthyronin und Reverse-Trijodthyronin $(\alpha=0,01)$, Calcium, Hydrogencarbonat, Harnsäure, $\gamma$-Globuline und $L$-Thyroxin $(\alpha=0,05)$ eine signifikante Unterscheidung. Keine signifikante Unterscheidung ist für die übrigen Parameter festzustellen.

Tab. 2. Konzentrationen in Zystenflüssigkeiten und Seren von Patienten mit gelben Strumapseudozysten.

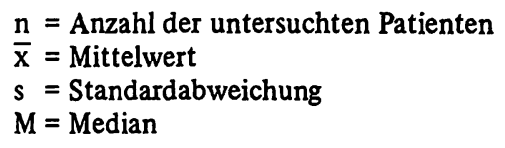

\begin{tabular}{|c|c|c|c|c|c|c|c|c|}
\hline \multirow[t]{2}{*}{ Stoff } & \multirow[t]{2}{*}{$\begin{array}{l}\text { Meß- } \\
\text { größe }\end{array}$} & \multirow[t]{2}{*}{$\mathrm{n}$} & \multicolumn{3}{|l|}{ Zyste } & \multicolumn{3}{|l|}{ Serum } \\
\hline & & & $\overline{\mathbf{x}}$ & $\pm s$ & $\mathbf{M}$ & $\overline{\mathbf{x}}$ & $\pm s$ & M \\
\hline Bilirubin & $\mathrm{mmol} / 1$ & 10 & 12,5 & 11,6 & 8,6 & 6,8 & 4,6 & 5,6 \\
\hline Glucose & $\mathrm{mmol} / \mathrm{l}$ & 9 & 5,42 & 1,39 & 6,17 & 4,72 & 1,26 & 5,12 \\
\hline Triglyceride & $\mathrm{mmol} / \mathrm{l}$ & 9 & 0,41 & 0,19 & 0,39 & 1,96 & 1,40 & 1,39 \\
\hline Cholesterin & $\mathrm{mmol} / \mathrm{l}$ & 8 & 2,55 & 1,03 & 2,26 & 6,10 & 1,60 & 6,12 \\
\hline Magnesium & $\mathrm{mmol} / \mathrm{l}$ & 8 & 0,86 & 0,08 & 0,82 & 0,99 & 0,12 & 0,95 \\
\hline Calcium & $\mathrm{mmol} / \mathrm{l}$ & 10 & 2,27 & 0,47 & 2,10 & 2,45 & 0,16 & 2,47 \\
\hline anorg. Phosphor & $\mathrm{mmol} / \mathrm{l}$ & 8 & 0,71 & $0 ; 39$ & 0,87 & 1,13 & 0,16 & 1,07 \\
\hline Eisen & $\mu \mathrm{mol} / 1$ & 12 & 20,2 & 10.6 & 17,5 & 17,5 & 6,8 & 17,8 \\
\hline Kupfer & $\mu \mathrm{mol} / \mathrm{l}$ & 8 & 14,2 & 5,7 & 12,3 & 23,2 & 7,0 & 22,4 \\
\hline Kalium & $\mathrm{mmol} / \mathrm{l}$ & 10 & 4,00 & 0,60 & 3,80 & 4,40 & 0,40 & 4,50 \\
\hline Natrium & $\mathrm{mmol} / \mathrm{l}$ & 10 & 135,2 & 6,7 & 137,5 & 141,7 & 3,2 & 141,5 \\
\hline Chlorid & $\mathrm{mmol} / \mathrm{l}$ & 10 & 104,0 & 4,5 & 106,0 & 104,4 & 2,5 & 103,5 \\
\hline Hydrogencarbonat & $\mathrm{mmol} / \mathrm{l}$ & 10 & 22,4 & 4,4 & 22,5 & 22,7 & 2,6 & 23,0 \\
\hline Harnstoff & $\mathrm{mmol} / \mathrm{l}$ & 10 & 4,82 & 1,17 & 5,33 & 4,61 & 1,05 & 4,66 \\
\hline Creatinin & $\mu \mathrm{mol} / 1$ & 10 & 79,6 & 17,7 & 88,4 & 88,4 & 17,7 & 88,4 \\
\hline Harnsäure & $\mu \mathrm{mol} / 1$ & 12 & 273,6 & 59,5 & 267,7 & 279,6 & 65,4 & 273,6 \\
\hline Protein & $\mathrm{g} / 1$ & 10 & 54,0 & 9,4 & 56,50 & 75,1 & 9,4 & 74,5 \\
\hline Albumine & $\mathrm{g} / 1$ & 9 & 31,4 & 10,32 & 32,85 & 43,1 & 4,4 & 42,8 \\
\hline$\alpha_{1}$-Globuline & $\mathrm{g} / 1$ & 9 & 2,60 & 0.87 & 2,40 & 2,96 & 0,7 & 2,84 \\
\hline$\alpha_{2}$-Globuline & $\mathrm{g} / 1$ & 9 & 3,54 & 1,95 & 3,11 & 7,46 & 2,6 & 7,1 \\
\hline$\beta$-Globuline & $g / 1$ & 9 & 5,48 & 1,65 & 5,67 & 8,5 & 1,7 & 8,4 \\
\hline$\gamma$-Globuline & $\mathrm{g} / \mathrm{l}$ & 9 & 8,27 & 2,49 & 7,44 & 13,6 & 2,7 & 13,7 \\
\hline$\alpha_{1}+\alpha_{2}$-Globuline & $\mathrm{g} / 1$ & 9 & 6,14 & 2,69 & 5,26 & 10,4 & 3,2 & 9,9 \\
\hline Alkalische Phosphatase & $\mathrm{U} / 1$ & 9 & 20,2 & 27,3 & 13,0 & 35,5 & 15,6 & 31,0 \\
\hline Saure Phosphatase & $\mathrm{U} / 1$ & 9 & 3,8 & 3,5 & 2,4 & 8,9 & 2,9 & 8,6 \\
\hline Alaninaminotransferase & $\mathrm{U} / 1$ & 9 & 4,2 & 1,9 & 4,0 & 6,7 & 5,1 & 5,0 \\
\hline Aspartataminotransferase & $\mathrm{U} / \mathrm{l}$ & 9 & 6,0 & 1,8 & 6,0 & 10,2 & 5,2 & 9,0 \\
\hline Lactatdehydrogenase & $\mathrm{U} / \mathrm{l}$ & 9 & 121,2 & 46,3 & 118,0 & 213,3 & 61,7 & 200,0 \\
\hline$\alpha$-Hydroxybutyratdehydrogenase & $\mathrm{U} / 1$ & 10 & 66,4 & 17,8 & 64,5 & 128,4 & 29,8 & 123,0 \\
\hline$\alpha$-Amylase & $\mathrm{U} / 1$ & 9 & 805,6 & 513,8 & 620,0 & 568,9 & 519,0 & 400,0 \\
\hline$\gamma$-Glutamyltranspeptidase & $\mathrm{U} / \mathrm{I}$ & 9 & 10,3 & 13,8 & 5,9 & 24,7 & 24,3 & 16,0 \\
\hline Cholinesterase & $\mathrm{U} / \mathrm{l}$ & 9 & 2210,0 & 1181,0 & 1950,0 & 6801,0 & 1477,0 & 6679,0 \\
\hline Proteingebundenes Jod & $\mathrm{nmol} / \mathrm{l}$ & 11 & 425,5 & 157,6 & 346,7 & 472,8 & 94,7 & 441,3 \\
\hline$L$-Trijodthyronin & $\mu \mathrm{g} / \mathrm{l}$ & 15 & 2,60 & 2,08 & 1,45 & 1,30 & 0,27 & 1,20 \\
\hline Reverse-Trijodthyronin & $\mathrm{ng} / \mathrm{l}$ & 15 & 240 & 190 & 170 & 180 & 30 & $180^{\circ}$ \\
\hline$L$-Thyroxin & $\mu \mathrm{g} / \mathrm{l}$ & 15 & 90,4 & 46,2 & 84,0 & 77,3 & 24,5 & 80 \\
\hline Insulin & $\mathrm{mU} / \mathrm{l}$ & 19 & 8,6 & 7,7 & 7,3 & 11,1 & 12,6 & 8,0 \\
\hline Wachstumshormon & $\mu \mathrm{g} / \mathrm{l}$ & 22 & 1,9 & 1,9 & 1,1 & 2,9 & - 2,2 & 2,4 \\
\hline
\end{tabular}




\section{Diskussion}

Alle im Serum überprüften Substanzen finden sich auch in den Zystenflüssigkeiten. In der braunen Zystenflüssigkeit sind die Werte meist signifikant höher und ohne Korrelation zu den Serumwerten, in der gelben Zystenflüssigkeit meist niedriger und mit den Serumwerten gut korreliert. Für die unterschiedlichen Konzentrationen in den Zystenflüssigkeiten sind verschiedene Faktoren verantwortlich. Eine wesentliche Rolle kommt den Austauschvorgängen zwischen Blut und Zystenflüssigkeit zu: Funktionsuntersuchungen (22) mit ${ }^{131} \mathrm{Jod}$ und $99 \mathrm{~m} \mathrm{Tc}$ Pertechnetat p.o. zeigen innerhalb von 2 Stunden einen guten Übertritt in gelbe und braune Zystenflüssigkeiten bei guter Korrelation mit den Serumwerten. I.v. injiziertes ${ }^{51} \mathrm{Cr}$-Albumin diffundiert innerhalb von 24 Stunden in gelbe Zystenflüssigkeit sehr gut, in braune nur in geringem Ausmaß. $\left[{ }^{125} \mathrm{~J}\right]-L$-Trijodthyronin, ${ }^{51} \mathrm{Cr}$-Natriumchromat und ${ }^{51} \mathrm{Cr}$-Albumin wandern aus gelben Pseudozysten innerhalb von 24 Stunden sehr gut, aus braunen Zysten dagegen nur in geringer Menge ab. Kolloidales ${ }^{198} \mathrm{Au}$ mit einer Teilchengröße von $5 \mathrm{~nm}$ wurde bei einer von vier gelben Zysten und bei drei von sechs braunen Zysten jeweils in geringer Menge abtransportiert, bei der gelben Pseudozyste konnte szintigraphisch ein lymphatischer Abtransport dokumentiert werden.

\section{Braune Pseudozysten}

Braune Pseudozysten entstehen durch Hämorrhagie in meist degenerativ vorgeschädigtes Schilddrüsengewebe (23). Der Zysteninhalt besteht daher aus den Bestandteilen des Blutes, zugrundegegangenem Schilddrüsengewebe, Kolloid aus aufgebrochenen Follikeln und Lymphe.

Zytologisch finden sich massenhaft Erythrocyten, sowie Granulocyten, Lymphocyten, Monocyten, Phagocyten und Thyreocyten, überwiegend mit degenerativen Veränderungen.

Durch autolytische Vorgänge werden vor allem Eisen, Lactatdehydrogenasen, $\alpha$-Hydroxybutyratdehydrogenasen, Aspartataminotransferase und saure Phosphatasen frei. Auf Grund der schlechten Austauschvorgänge zwischen Zystenflüssigkeit und Blut kommt es zu einer Konzentration dieser Substanzen und auch des Proteins sowie der einzelnen Proteinfraktionen. Aus hohen Werten der Lactatdehydrogenase in der Zystenflüssigkeit kann nicht auf Malignität geschlossen werden. Metabolische Vorgänge sind für die Erhöhung der Harnnsäure und für die Verminderung von Insulin, Glucose, Hydrogencarbonat und Triglyceriden, sowie für unterschiedliche Enzymkonzentrationen verantwortlich. Hämolytisches Serum baut markiertes Insulin rasch ab (24). Wie in länger gelagerten Blutkonserven ist die Glucoseverminderung durch anaerobe Glykolyse in den Erythrocyten (25) und die Verminderung der Triglyceride durch Hydrolyse und Oxidation (26) bedingt. Dagegen fehlt eine Kaliumerhöhung in der
Zystenflüssigkeit, woraus auf eine gute Diffusion für Kalium, wahrscheinlich auch für Kupfer, Natrium, Chlorid, Harnstoff und Creatinin zu schließen ist.

Ursache für die z.T. hohe Konzentration von proteingebundenem Jod, $L$-Trijodthyronin, Reverse-Trijodthyronin und $L$-Thyroxin sind ein Übertritt von Hormonen und Jodproteinen aus den durch Blutung zerstörten Follikeln und Lymphgefäßen, die hohe Proteinkonzentration der Zystenflüssigkeit und ein nur geringer $\mathrm{Ab}$ transport, sowie eine Inkretion von Schilddrüsenhormonen bzw. Hormonvorstufen durch das die Pseudozyste in unterschiedlichem Ausmaß wandbildend umgebende Schilddrüsengewebe. Eine derartige Inkretion von Tyrosinen konnten wir radiochromatographisch nachweisen (Riccabona, G. \& Galvan, G., in Vorbereitung). Inwieweit Monodejodierungsvorgänge an dem verhältnismäßig hohen Anteil von $L$-Trijodthyronin und Reverse-Trijodthyronin beteiligt sind, ist nicht zu unterscheiden.

Bei wiederholten Punktionen brauner Pseudozysten sinken alle erhöhten Werte allmählich ab, die Farbe wird zunehmend hellbraun bis gelb, soferne es nicht zu neuen Blutungen kommt.

\section{Gelbe Pseudozysten}

Für die Bildung von gelben Pseudozysten werden eine Follikeldilatation und Follikelkonfluenz durch Kolloidresorptionsstörungen und Lymphstauung, andererseits eine Entstehung aus Blutungspseudozysten durch allmähliche Resorption des Inhaltes angenommen (23).

Zytologisch ist die gelbe Zystenflüssigkeit sehr zellarm, auch im Sediment finden sich meist nur wenige Phagocyten und Thyreocyten, so daß Veränderungen infolge metabolischer oder autolytischer Prozesse durch die in der Zystenflüssigkeit vorhandenen Zellen unerheblich sind. Fast sämtliche untersuchten Substanzen, insbesondere auch das Protein, sind in der Zystenflüssigkeit signifikant gegenüber den Serumwerten erniedrigt, bei zumeist guter Korrelation. Dies ist als Folge der sehr guten Austauschvorgänge Zwischen Zystenflüssigkeit und Blut zu werten.

Stärkere Abweichungen in einzelnen gelben Pseudozysten gegenüber den Blutwerten liegen vor allem für Substanzen vor, die kurzfristig stärkeren Schwangungen im Blut unterworfen sind, wie für Wachstumshormon, Insulin, Cholesterin, Triglyceride und Glucose. Die zwar nicht signifikanten, aber bei vielen gelben Zysten erhöhten $L$-Trijodthyronin- und Reverse TrijodthyroninWerte sind wiederum durch Inkretion und/oder Monodejodierung zu erklären. Bis zu 6 Punktionen gelber Zysten in mehrtägigen Abständen ergaben eine annähernd gleichbleibende Konzentration aller Substanzen.

\section{Folgerung}

1. Zystenflüssigkeiten und Seren weisen eine qualitativ ähnliche Zusammensetzung auf. 
2. In gelben Zystenflüssigkeiten sind fast alle Substanzen niedriger als in den zugehörigen Seren bei guter Korrelation mit den Serumwerten, bedingt durch einen guten Austausch mit dem Blut. Braune Zysten: flüssigkeiten dagegen zeigen einen nur geringen Austausch mit dem Blut, durch den hohen Zellgehalt kommt es zu autolytisch und metabolisch bedingten beträchtlichen Konzentrationsunterschieden einiger Substanzen in Zystenflüssigkeiten gegenüber den Seren. Hohe Werte für die Lactatdehydrogenase in der Zystenflüssigkeit können nicht als Hinweis für Malignität gewertet werden.

Die für gelbe und braune Zysten nachweisbaren Austauschvorgänge mit dem Blut erklären den verhältnismäßig geringen Anteil dauerhafter Entleerungen von Strumazysten durch therapeutische Feinnadelpunktion von $30 \%$ bei gelben und $43 \%$ bei braunen Zysten (27).

3. Die höheren Werte für $L$-Trijodthyronin, ReverseTrijodthyronin und $L$-Thyroxin in braunen, sowie in einigen gelben Zysten können auch durch eine direkte Abgabe von Schilddrüsenhormonen aus Schilddrüsengewebe in der Zystenwand zustāndekommen. Bei erhaltener Regulationsfähigkeit der Struma ist ein Ubertritt der Hormone aus der Zyștenflüssigkeit in das Blut belanglos. Bei zystischer Degeneration automomer Strumen können aber auf diesem Weg klinisch relevante Hormonmengen in das Blut gelangen.

\section{Literatur}

1. Galvan, G. (1977), Münch. Med. Wochenschr. 119, 229-232.

2. Müller, H. Ch. \& Zita, G. (1973), Radiobiol.-Radiother. S, 531-534.

3. Rilke, F., Colombo, F. \& Pecchiai, L. (1956), Virchows Archiv 328, 552-564.

4. Galvan, G. (1973), Dtsch. Med. Wochenschr. 98, 21072110.

5. Technicon Auto Analyzer II (1973), Clinicai Method No Se 2-0018FF3.

6. Technicon Auto Analyzer II (1972), Clinical Method No 26 a.

7. Dannenberg, E. (1956), Ärztliches Labor 2, 223-225.

8. Technicon Auto Analyzer II (1972), Clinical Method No. 25.

9. Holy, H. W. (1969), Technicon Internat. Symposium London, 37-44.

10. Skeggs, L. T. \& Holzstrasser, H. (1964), Clin. Chem. 10, 918-936.

11. Müller-Plathe, O. (1973), in: Klinische Chemie in Einzeldarstellungen, Georg Thieme Stuttgart 172-173.

12. March, W. H., Fingerhut, B. \& Miller, H. (1965), Clin. Chem. $11,624-627$.

13. Chasson, A. L., Grady, H. J. \& Stanley, M. A. (1961), Am. J. Clin. Pathol 35, 83-87.

14. Weichselbaum, T. E., Technicon Auto Analyzer-Methodology N-14b.
15. Eppendorf Analysensysteme, Arbeitsvorschrift AV 732/722 A.

16. Richterich, R. (1968), in: Klinische Chemie, 2. Aufl., S. Karger/Basel, S. 406-408.

17. Gibitz, H. J. \& Stöllinger, Ch. (1970), Technicon Symposium 1970, Bad Homburg.

18. Sachs, L. (1972), Statistische Auswertungsmethoden, S. 244-246, Springer Verlag Berlin.

19. Sachs, L. (1972), Statistische Auswertungsmethoden, S. 309-311, Springer Verlag Berlin

20. Dixon, W. J. (1975), BMDP Biomedical Computer Programs, S. 657-681, Univèrsity of California Press, Berkeley.

21. Dixon, W. J., Massey, F. J. (1969), Introduction to statistical analysis, S. 545-549, Mc. Graw Hill Tokyo.

22. Galvan, G. \& Maier, F. (1976), 14. Internationale Tagung der Gesellschaft für Nuklearmedizin Berlin, Abstract 102.

23. Wegelin, C. (1926), in: Handbuch der Speziellen Pathologischen Anatomie und Histologie, S. 172-228, Springer Verlag Berlin.

24. Pfeiffer, E. F. (1969), in: Handbuch des Diabetes mellitus, S. 256, J. F. Lehmanns Verlag München.

25. Blum, K. U. (1960), Bibl. Haemat. 12, 20-63.

26. Elmendorff, H. \& Vallee, D. (1964), Blut 10, 61-67.

27. Galvan, G. \& Maurer, H. (1977), Dtsch. Med. Wochenschr. $102,829-830$.

Dr. Günther Galvan,

Nuklearmedizinische Station und

Prim. Dr. Hansjörg Gibitz, Dr. Dieter Hauch,

Zentrallaboratorium der Landeskrankenanstalten Salzburg,

Franz Maier,

Zentrum für elektronische Datenverarbeitung der Universität Salzburg,

A-5020 Salzburg 